\title{
AVALIAÇÃO DO PROCESSO DE CLARIFICAÇÃO DA ÁGUA BRUTA POR MEIO DE DIAGRAMAS DE COAGULAÇÃO, EMPREGANDO A ASSOCIAÇÃO Moringa oleifera Lam E CLORETO FÉRRICO
}

Karina Cardoso Valverde ${ }^{1}$

Onélia Aparecida Andreo dos Santos ${ }^{2}$

Rosângela Bergamasco ${ }^{3}$

RESUMO: Este trabalho propõe avaliar o processo de coagulação/floculação e sedimentação, por meio de diagramas de coagulação, utilizando a associação dos coagulantes natural: Moringa oleifera Lam (MO) em pó e químico: cloreto férrico. Os ensaios foram realizados em Jar Test, com água bruta proveniente da bacia do Rio Pirapó, Maringá, PR, variando-se o pH de coagulação no intervalo de 4 a 10; e as dosagens de coagulantes natural e químico, a fim de verificar a eficiência de remoção dos parâmetros de qualidade cor aparente, turbidez e compostos com absorção em UV $\mathrm{UV}_{254 \mathrm{~nm}}$. Após os ensaios, amostras de água tratada foram coletadas para avaliar a eficiência do processo. Constatou-se que os diagramas de coagulação são ferramentas importantes,

\footnotetext{
${ }^{1}$ Engenheira Química, Acadêmica de Pós-graduação em Engenharia Química (Doutorado), Universidade Estadual de Maringá (UEM), Maringá, PR, Bolsista CAPES. ka.cc@bol.com.br

${ }^{2}$ Docente do Curso de Engenharia Química, Universidade Estadual de Maringá (UEM), Maringá, PR, Bolsista de Produtividade em Pesquisa do CNPq. onelia@deq.uem.br

${ }^{3}$ Docente do Curso de Engenharia Química, Universidade Estadual de Maringá (UEM), Maringá, PR, Bolsista de Produtividade em Pesquisa CNPq. rosangela@deq.uem.br
} 
pois possibilitam o desenvolvimento de ensaios nas condições ótimas, em função das características da água bruta. Foram obtidas redução de até $50 \%$ na dosagem do cloreto férrico, sem comprometer a qualidade da água produzida. Assim, a utilização de MO pode desempenhar um papel-chave na diminuição da quantidade requerida de coagulante químico utilizada no tratamento de água, além de ser mais compatível com as questões ambientais atuais. Além disso, a associação dos coagulantes apresentam um efeito sinérgico superior à utilização do pó de sementes de MO como único coagulante.

Palavras-chave: Diagramas de coagulação. Moringa oleifera Lam. Cloreto férrico.

\section{INTRODUÇÃO}

Mesmo que uma água bruta visualmente não apresente indicações de contaminação, não se pode assegurar sua qualidade física, química e microbiológica, sendo necessário um tratamento adequado para a obtenção de água potável. Dentre os tratamentos de água, a adição de coagulantes tem sido utilizada com sucesso.

Em relação aos coagulantes químicos, o cloreto férrico é representado pela fórmula molecular $\mathrm{FeCl}_{3} .6 \mathrm{H}_{2} \mathrm{O}$ e produz bons flocos no intervalo de $\mathrm{pH}$ entre 5 e 11 (SPINELLI, 2001).

A Moringa oleifera Lamarck (Lam) destaca-se como um coagulante natural. Essa planta apresenta a vantagem de adaptar-se em locais com pluviometria baixa e climas quentes e não ter exigências quanto ao tipo de solo (GIDDE et al., 2012).

As sementes de Moringa oleifera Lam (MO) apresentam o melhor potencial de coagulação/floculação (NWAIWU et al., 2012) do que as demais partes da planta, tais como folhas e vagens. Poumaye et al. (2012) avaliaram que esse biopolímero pode ser usado na clarificação da água com eficiência elevada.

Apesar de haver muitos estudos no sentido de isolar e caracterizar o princípio ativo coagulante encontrado nas sementes da $\mathrm{MO}$, supõe-se que essas são utilizadas na clarificação de água devido à presença de uma proteína coagulante catiônica solúvel 
capaz de reduzir a turbidez da água tratada (PRITCHARD et al., 2010; SANTOS et al., 2011; GIDDE et al., 2012).

Até o momento, nenhuma evidência foi encontrada de que as sementes possam causar efeitos secundários nos seres humanos, especialmente com as baixas doses necessárias para o tratamento da água (LEA, 2010; SANTOS et al., 2011).

Dessa forma, pode-se afirmar que o tratamento da água com a MO não apresenta risco à saúde (VALVERDE et al., 2013), já que além de atuar como agente clarificante de águas turvas e coloridas, também possuem inúmeros usos na alimentação humana (CARDOSO et al., 2008). Segundo Lea (2010) e Nwaiwu et al. (2012), a MO não é tóxica.

De acordo com Vijayaraghavan, Sivakumar e Vimal Kumar (2011), há escassez de estudos abrangentes que comparem a eficiência da utilização da associação dos coagulantes natural e químico.

Abaliwano et al. (2008) afirmam que, o uso de MO poderia conduzir a uma redução de $50 \%$ na utilização de produtos químicos inorgânicos. Desta forma, uma pequena dosagem desse coagulante natural, que é um produto renovável e biodegradável, pode reduzir o consumo de coagulantes químicos, tais como o cloreto férrico.

Uma forma bastante interessante a fim de definir a dosagem ótima de coagulante e o pH adequado para o processo de coagulação/floculação e sedimentação fundamenta-se na realização de ensaios realizados em Jar Test, que ocasionem a construção de diagramas de coagulação.

A utilização de ferramentas matemáticas capazes de definir as melhores condições a partir de um conjunto de variáveis é de extrema importância para obtenção das condições otimizadas do processo (VALVERDE et al., 2013).

Assim, este estudo propõe avaliar o processo de coagulação/floculação e sedimentação, por meio de diagramas de coagulação, utilizando a associação dos coagulantes $\mathrm{MO}$ e cloreto férrico.

\section{MATERIAIS E MÉTODOS}


Os ensaios de coagulação/floculação foram realizados no Laboratório de Gestão, Controle e Preservação Ambiental, do Departamento de Engenharia Química, da Universidade Estadual de Maringá, utilizando a água bruta proveniente da bacia do rio Pirapó, Maringá, PR, Brasil, coletada na Estação de Tratamento de Água (ETA) da cidade (Sanepar).

Para a preparação da solução padrão do coagulante cloreto férrico, foi considerada uma concentração de $1 \% \mathrm{~m} / \mathrm{v}$. Para a obtenção do coagulante em pó de $\mathrm{MO}, 5 \mathrm{~g}$ de sementes foram descascadas, trituradas em liquidificador (NL-41 Mondial) e secas em estufa com circulação e renovação de ar (Digital Timer SX CR/42) a $40^{\circ} \mathrm{C}$ até peso constante (AMAGLOH e BENANG, 2009).

Os ensaios de clarificação da água foram realizados em Jar Test Nova Ética Modelo 218/LDB06 de seis provas em recipientes com $400 \mathrm{~mL}$ de água bruta, mantidas na temperatura de $25,0 \pm 3,0^{\circ} \mathrm{C}$.

As condições de operação ótimas foram determinadas experimentalmente em Jar Test, especificamente para a associação dos coagulantes MO e cloreto férrico, e são: 100 rpm durante 3 min para propiciar a mistura rápida, 30 rpm durante 15 min para propiciar a mistura lenta e 15 min de sedimentação.

Após o processo, a amostra de água tratada foi retirada de cada um dos recipientes, contando com o auxílio de uma pipeta graduada de $20 \mathrm{~mL}$.

Os diagramas de coagulação foram obtidos por meio da realização de ensaios de coagulação/floculação e sedimentação, avaliando-se a eficiência de remoção de cor aparente, turbidez e compostos com absorção em $U_{254 \mathrm{~nm}}$ em função da variação do pH de coagulação e da dosagem dos coagulantes na associação. Estes foram construídos no programa 3DField 3.5.3.0.

$\mathrm{O}$ pH utilizado nos ensaios foi ajustado na faixa compreendida entre 4 e 10, com solução de hidróxido de sódio $(\mathrm{NaOH}) 0,1 \mathrm{~N}$ e $1 \mathrm{~N}$; e ácido clorídrico $(\mathrm{HCl}) 0,1 \mathrm{~N}$ e $1 \mathrm{~N}$.

As dosagens máximas, consideradas ótimas, adicionadas nos ensaios foram adaptadas, baseado em dados de turbidez inicial (BAGHVAND et al., 2010; JOSHUA e VASU, 2013). As dosagens dos coagulantes utilizadas estão apresentadas na Tabela 1. 
Tabela 1. Dosagem dos coagulantes na associação

\begin{tabular}{|c|c|c|c|c|c|c|c|c|c|c|c|c|}
\hline \multicolumn{2}{|c|}{ Ponto } & 1 & 2 & 3 & 4 & 5 & 6 & 7 & 8 & 9 & 10 & 11 \\
\hline \multicolumn{2}{|c|}{$\begin{array}{l}\% \text { Coagulante } \\
\text { (Cloreto / MO) }\end{array}$} & $\begin{array}{c}0 / \\
100 \%\end{array}$ & $\begin{array}{l}10 / \\
90 \%\end{array}$ & $\begin{array}{l}20 / \\
80 \%\end{array}$ & $\begin{array}{l}30 / \\
70 \%\end{array}$ & $\begin{array}{l}40 / \\
60 \%\end{array}$ & $\begin{array}{l}50 / \\
50 \%\end{array}$ & $\begin{array}{l}60 / \\
40 \%\end{array}$ & $\begin{array}{l}70 / \\
30 \%\end{array}$ & $\begin{array}{l}80 / \\
20 \%\end{array}$ & $\begin{array}{l}90 / \\
10 \%\end{array}$ & $\begin{array}{c}100 / \\
0 \%\end{array}$ \\
\hline \multirow{2}{*}{$\begin{array}{l}\text { Dosagem } \\
\left(\mathrm{mg}^{-1} \mathrm{~L}^{-1}\right)\end{array}$} & Cloreto & 0 & 1 & 2 & 3 & 4 & 5 & 6 & 7 & 8 & 9 & 10 \\
\hline & MO & 50 & 45 & 40 & 35 & 30 & 25 & 20 & 15 & 10 & 5 & 0 \\
\hline
\end{tabular}

A caracterização da água bruta foi realizada por meio dos parâmetros: cor aparente e compostos com absorção em $U^{254 n m}$ (espectrofotômetro DR 5000 Hach), turbidez (turbidímetro 2100P Hach), sólidos dissolvidos totais (SDT) (APHA, 1995) e pH (pHmetro Thermo-Scientific VSTAR92 Orion Versastar).

\section{RESULTADOS E DISCUSSÕES}

A Tabela 2 apresenta os resultados da caracterização da água bruta utilizada nos ensaios de coagulação/floculação e sedimentação.

Tabela 2. Caracterização da água bruta

\begin{tabular}{|c|c|c|c|c|c|}
\hline Parâmetro de qualidade & Cor aparente & Turbidez & UV $_{254 \mathrm{~nm}}$ & SDT & pH \\
\hline Água bruta & $426 \mathrm{uH}$ & $74,9 \mathrm{NTU}$ & $0,294 \mathrm{~cm}^{-1}$ & $159,7 \mathrm{mg}_{\mathrm{L}} \mathrm{L}^{-1}$ & 7,940 \\
\hline
\end{tabular}

Nas Figuras 1 (A), (B) e (C) constam os diagramas de coagulação avaliando a eficiência de remoção dos parâmetros de qualidade cor aparente, turbidez e compostos com absorção em $U_{254 n m}$. 
(A)

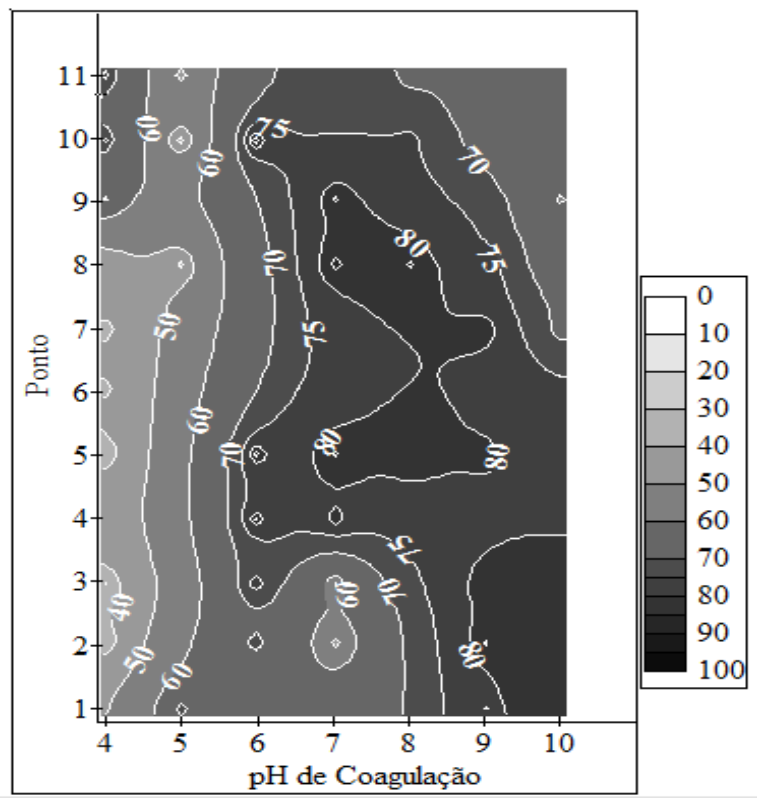

(B)

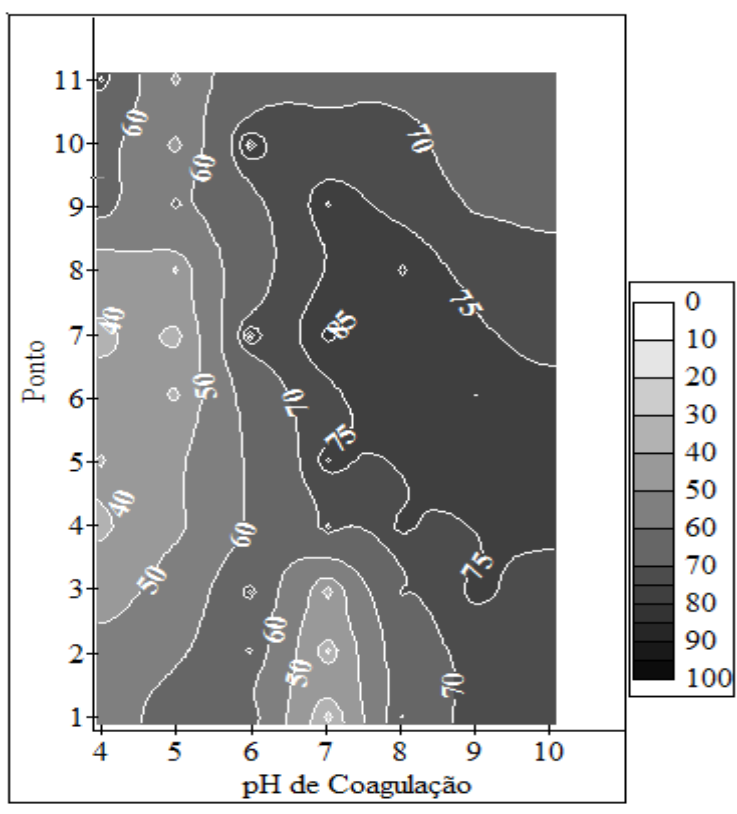

(C)

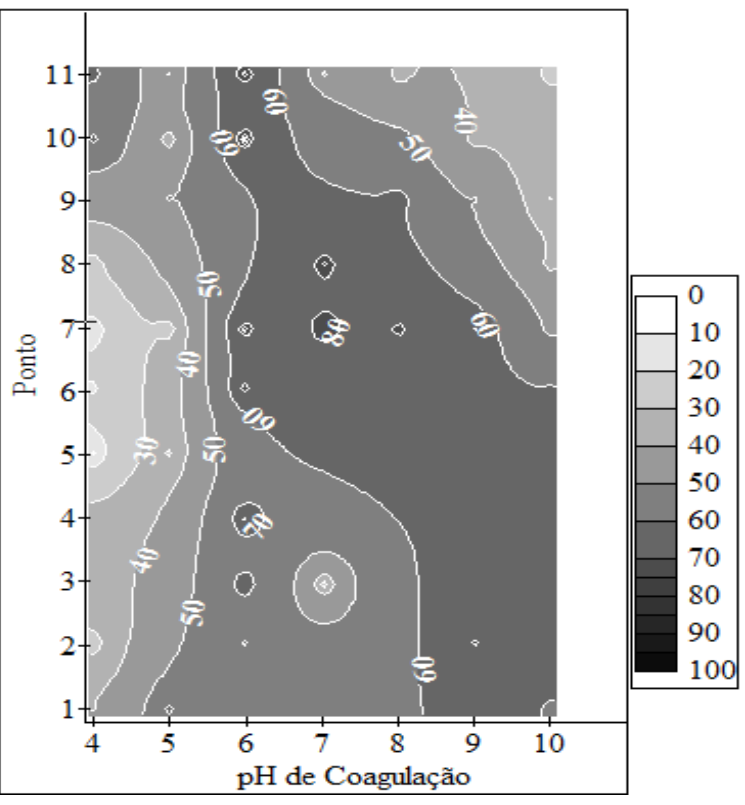

Figura 1. Diagramas de coagulação para a associação dos coagulantes $M O$ e cloreto férrico contendo curvas de remoção de: (A) cor aparente; (B) turbidez; (C) compostos com absorção em $U^{254 n m}$

De um modo geral, baseado nos três diagramas de coagulação, percebeu-se que a faixa de $\mathrm{pH}$ compreendida entre 4 e 5 apresenta remoções inferiores aos demais pHs de coagulação. $\mathrm{O}$ intervalo de pH entre 6 e 10 apresentou remoções mais eficientes, o que já 
era esperado, já que Spinelli (2001) afirma que, o cloreto férrico tende a produzir bons flocos no intervalo de $\mathrm{pH}$ entre 5 e 11.

A avaliação dos três parâmetros de qualidade nos pH de coagulação 6 e 7, por meio de análise estatística (delineamento inteiramente casualizado), demonstram que não há diferença significativa nas eficiências de remoção obtidas quando se utiliza a dosagem de $4 \mathrm{mg} / \mathrm{L}^{-1}$ cloreto férrico/30 mg. $\mathrm{L}^{-1} \mathrm{MO}$ (ponto 5 ) até $9 \mathrm{mg} / \mathrm{L}^{-1}$ cloreto férrico $/ 5 \mathrm{mg} \cdot \mathrm{L}^{-1} \mathrm{MO}$ (ponto 10).

Desse modo, é possível obter uma redução de até $50 \%$ no consumo do cloreto férrico com o aumento da dosagem de $\mathrm{MO}$, sem afetar a eficiência do processo, o que corrobora a afirmação de Abaliwano et al. (2008) que destacam que, o uso de MO poderia conduzir a uma redução de 50\% na utilização de agentes químicos inorgânicos.

Para o $\mathrm{pH}$ de coagulação 8, pode-se afirmar que a dosagem de $3 \mathrm{mg} / \mathrm{L}^{-1}$ cloreto férrico/35 mg. $\mathrm{L}^{-1} \mathrm{MO}$ (ponto 4) até $7 \mathrm{mg} / \mathrm{L}^{-1}$ cloreto férrico/15 mg.L $\mathrm{L}^{-1} \mathrm{MO}$ (ponto 8) são consideradas estatisticamente iguais, o que resulta na diminuição de até $40 \%$ na utilização do coagulante químico.

Todavia, quando se avalia o residual, observa-se médias em torno de $60 \mathrm{uH}$ para cor aparente, 16,00 NTU para turbidez e 0,080 $\mathrm{cm}^{-1}$ para compostos com absorção em $U V_{254 n m}$, sendo esses considerados altos, em comparação aos apresentados Portaria nํ․ 2914 do Ministério da Saúde (BRASIL, 2011). Então a associação dos coagulantes utilizada no processo de coagulação/ floculação e sedimentação não produz água potável. Sendo assim, é importante acrescentar a etapa de filtração para melhorar a qualidade da água tratada.

No intervalo de pH entre 9 e 10, não houve variações expressivas nas eficiências de remoção dos parâmetros de qualidade até o ponto 7 , que utiliza $6 \mathrm{mg} / \mathrm{L}^{-1}$ cloreto férrico/20 mg. $\mathrm{L}^{-1} \mathrm{MO}$.

\section{CONCLUSÕES}


O diagrama de coagulação é uma ferramenta bastante interessante a fim de determinar as condições ótimas de trabalho, em função das características da água de estudo.

Pode-se concluir que a associação dos coagulantes $\mathrm{MO}$ e cloreto férrico apresentam um efeito sinérgico superior à utilização do pó integral de sementes de MO como único coagulante. A utilização da MO contribui significativamente para a redução da quantidade de coagulantes químicos aplicado ao processo e é mais compatível com as questões ambientais atuais.

\section{AGRADECIMENTOS}

Os autores agradecem a CAPES pelo suporte financeiro; a Universidade Federal de Sergipe (UFS), pela doação das sementes de MO; e a SANEPAR, pelas amostras de água bruta fornecidas.

\section{REFERÊNCIAS}

ABALIWANO, J. K.; GHEBREMICHAEL, K. A.; AMY, G. L. Application of the purified Moringa oleifera coagulant for surface water treatment. WaterMill Working Paper Series, n. 5, p. 1-19, 2008.

AMAGLOH, F. K.; BENANG, A. Effectiveness of Moringa oleifera seed as coagulant for water purification. African Journal of Agricultural Research, v. 4, n. 1, p. 119-123, feb. 2009.

APHA. American Public Health Association. Standard methods for the examination for water and wastewater. $19^{\text {th }}$ ed. Washington, 1995.

BAGHVAND, A.; ZAND, A. D.; MEHRDADI, N.; KARBASSI, A. Optimizing coagulation process for low to high turbidity waters using aluminum and iron salts. American Journal of Environmental Sciences, v. 6, n. 5, p. 442-448, 2010.

BRASIL. Ministério da Saúde. Portaria n. 2914, de 12 de dezembro de 2011. Dispõe sobre os procedimentos de controle e de vigilância da qualidade da água para consumo humano e seu padrão de potabilidade. Diário Oficial, Brasília, DF, 14 dez. 2011. Seção 1, p. 39. 
CARDOSO, K. C.; BERGAMASCO, R.; COSSICH, E. S.; MORAES, L. C. K. Otimização dos tempos de mistura e decantação no processo de coagulação/floculação da água bruta por meio da Moringa oleifera Lam. Acta Scientiarum Technology, Maringá, v. 3, n. 2, p. 193-198, 2008. doi:10.4025/actascitechnol.v30i2.5493.

GIDDE, M. R.; BHALERAO, A. R.; MALUSARE, C. N. Comparative study of different forms of Moringa oleifera extracts for turbidity removal. International Journal of Engineering Research and Development, v. 2, n.1, p. 14-21, jul. 2012.

JOSHUA, R.; VASU, V. Characteristics of stored rain water and its treatment technology using Moringa seeds. International Journal of Life Sciences Biotechnology and Pharma Research, India, v. 2, n. 1, p. 154-175, jan. 2013.

LEA, M. Bioremediation of turbid surface water using seed extract from Moringa oleifera Lam. (drumstick) tree. Current Protocols in Microbiology, Wiley Interscience, p. 1-14, feb. 2010. doi:10.1002/9780471729259.mc01g02s16.

NWAIWU, N. E; ZALKIFUL, M. A.; RAUFU I. A. Seeking an alternative antibacterial and coagulation agent for household water treatment. Journal of Applied Phytotechnology in Environmental Sanitation, Indonésia, v. 1, n. 1, p. 1-9, jan. 2012.

PRITCHARD, M.; CRAVEN, T.; MKANDAWIRE, T.; EDMONDSON, A. S.; O'NEILL, J. G. A comparison between Moringa oleifera and chemical coagulants in the purification of drinking water - An alternative sustainable solution for developing countries. Physics and Chemistry of the Earth, v. 35, n. 13-14, p. 798-805, jul. 2010.

doi:10.1016/j.pce.2010.07.014.

POUMAYE, N.; MABINGUI, J.; LUTGEN, P.; BIGAN, M. Contribution to the clarification of surface water from the Moringa oleifera: Case M'Poko river to Bangui, Central African Republic. Chemical Engineering Research and Design, v. 90, p. 2346-2352, dec. 2012. doi: 10.1016/j.cherd.2012.05.017.

SANTOS, W. R. dos; MATOS, D. B. de; OLIVEIRA, B. M.; SANTANA, T. M.; SANTANA, M. M. de; SILVA, G. F. da. Estudo do tratamento e clarificação de água com torta de sementes de Moringa oleifera Lam. Revista Brasileira de Produtos Agroindustriais, Campina Grande, v. 13, n. 3, p. 293-297, 2011.

SPINELLI, Viviane Aparecida. Quitosana: Polieletrólito Natural para o Tratamento de Água Potável. 2001. 134 p. Dissertação (Mestrado em Engenharia Ambiental) - Centro Tecnológico, Universidade Federal de Santa Catarina, Florianópolis, Santa Catarina, Brasil, 2001.

VALVERDE, K. C.; MORAES, L. C. K.; BONGIOVANI, M. C.; CAMACHO, F. P. BERGAMASCO, R. Coagulation diagram using the Moringa oleifera Lam and the 
aluminium sulphate, aiming the removal of color and turbidity of water. Acta Scientiarum Technology, Maringá, v. 5, n. 3, p. 485-489, jul./sep. 2013. doi:10.4025/actascitechnol.v35i3.12268.

VIJAYARAGHAVAN, G; SIVAKUMAR, T; VIMAL KUMAR, A. Application of plant based coagulants for waste water treatment. International Journal of Advanced Engineering Research and Studies, v. 1, n. 1, p. 88-92, oct./dec. 2011. 